\title{
How does ecological disturbance influence genetic diversity?
}

Sam C. Banks ${ }^{1,2}$

Geoffrey J. Cary ${ }^{1}$

Annabel L. Smith ${ }^{1,2}$

Ian Davies ${ }^{1}$

Don A. Driscoll ${ }^{1,2}$

A. Malcolm Gill ${ }^{1}$

David B. Lindenmayer ${ }^{1,2}$

Rod Peakall ${ }^{3}$

1 The Fenner School of Environment and Society, The Australian National University, Canberra, ACT, 0200, Australia

2. Australian Research Council Centre of Excellence for Environmental Decisions and the National Environmental Research Program Environmental Decisions Hub

${ }^{3}$ Evolution, Ecology and Genetics, Research School of Biology, The Australian National University, Canberra, ACT, 0200, Australia

Corresponding author: Banks, S.C. (sam.banks@anu.edu.au) 


\begin{abstract}
Environmental disturbance underpins the dynamics and diversity of many of the world's ecosystems, yet its influence on the patterns and distribution of genetic diversity is poorly appreciated. We argue here that disturbance history may be the major driver that shapes patterns of genetic diversity in many natural populations. We outline how disturbance influences genetic diversity through changes in both selective processes and demographically-driven, selectively-neutral processes. Our review highlights the opportunities and challenges presented by genetic approaches, such as landscape genomics, for better understanding and predicting the demographic and evolutionary responses of natural populations to disturbance. Developing this understanding is now critical as disturbance regimes are changing rapidly in a human-modified world.
\end{abstract}

\title{
Why should we consider disturbance as a driver of the distribution of genetic diversity?
}

Environmental disturbance underpins the dynamics and diversity of many of the world's ecosystems $[1,2]$. From tsunamis and large wild-land fires to footprints on intertidal mudflats, disturbances drive spatial and temporal variation in the abundance of species and the composition of communities [3-5]. Despite recognition that disturbance plays a key role in shaping biodiversity at the species and community levels $[2,6]$, its role as a driver of the patterns and distribution of genetic diversity (see Glossary) is relatively poorly understood. This is a major knowledge gap because genetic diversity has important consequences for all levels of biodiversity; it influences the fitness of individuals, the viability of populations, the adaptability of species to environmental change, the evolution of new species, the structure of communities, and the function of ecosystems [7-11]. Therefore, the interactions between disturbance and genetic diversity are likely to have pervasive ecological and evolutionary ramifications $[10,12]$. 
Improving our understanding of how disturbance influences the patterns of genetic diversity is critical for three reasons:

i. Disturbance patterns are changing rapidly in a human-modified world. Climate change is affecting the intensity and frequency of floods, hurricanes and extreme temperature events [13-16], fire regimes are being modified by human activities such as fire suppression and planned burning [17], and natural disturbances are interacting with novel anthropogenic disturbances [18]. Therefore, disturbance will increase in importance as a driver of the distribution of biodiversity across the genetic, species and community levels.

ii. The spatial and temporal patterns of genetic diversity can potentially tell us much about the demographic response of populations to disturbance. Genetic analyses have enabled new insights into the ecological consequences of major environmental changes such as habitat fragmentation, but such analyses have been less frequently applied in disturbance ecology. In particular, there has been limited evaluation of the potential for genetic analyses to improve biological inference under dynamic environmental and demographic conditions.

iii. Disturbance history may be the major driver that shapes the patterns of genetic diversity in many natural populations. This is because disturbance can cause variation in key demographic and biological processes that are known to influence the distribution of genetic diversity [19-22]. Therefore, studies that infer biological processes from genetic patterns, such as landscape genetics [23], must consider disturbance history alongside contemporary landscape patterns.

In this paper, we synthesise knowledge about how disturbance shapes genetic diversity. Our focus is on genetic diversity within species, and how it is distributed within and among individuals and populations in natural landscapes. We first define the key concepts of 
disturbance ecology and then identify how disturbance may influence genetic diversity via changes in both selective processes and demographically-driven, selectively-neutral processes. We then discuss the capacity of genetic analyses to inform our understanding of the biotic consequences of disturbance. Finally, we list the key questions that remain to be answered before we can more fully understand the interplay between disturbance and genetic diversity and how these interactions influence species conservation, community composition and evolution.

\section{What is ecological disturbance and why is it important?}

Ecological disturbances are commonly defined as "any relatively discrete event in time that disrupts ecosystem, community, or population structure and changes resources, substrate availability, or the physical environment" [24]. Disturbances are ubiquitous across the Earth's ecosystems. They occur from very fine spatial scales, such as trampling and wave-induced boulder turnover [25, 26], to large scales, such as landscape-wide fires and tsunamis [27, 28]. They can also occur over different temporal scales, as short 'pulse' disturbances (e.g. vegetation fires), as extended 'press' disturbances (e.g. grazing or browsing), or as 'ramp' disturbances that increase in intensity and resultant severity over time (e.g. drought effects on streams) [29]. Disturbances can have abiotic sources, such as hurricanes and volcanoes [30, 31], or biotic sources, such as grazing or pest outbreaks [32]. They can also have anthropogenic sources, such as logging [33]. Within the scope of this review, we do not consider disturbance to encompass long-term environmental changes such as climatic fluctuations or habitat clearing and fragmentation, which are also known to have genetic consequences $[34,35]$.

Large, severe disturbances are often portrayed as natural disasters, because they can threaten human life and have striking short-term effects on plant and animal populations [36]. However, disturbances play crucial roles in the formation and maintenance of ecological 
communities $[6,37]$ and many species are dependent on post-disturbance conditions for survival [38]. Disturbances occur in the context of a disturbance regime, which describes the type, season, frequency and intensity of disturbance at a specific location [39, 40]. Disturbance regimes can have important influences on the evolution of species [41] and the structure and diversity of ecosystems [6]. Changing disturbance regimes pose direct risks for the extinction of species [42], and such changes are occurring worldwide [13-15, 43]. Thus, building an understanding of the biotic consequences of disturbance and changing disturbance regimes is increasingly important if we are to anticipate future biodiversity scenarios [2].

\section{How does disturbance influence genetic diversity through selectively-neutral}

\section{demographic processes?}

To set the context for understanding how disturbance shapes the distribution of genetic diversity, it is important to understand the environmental and demographic changes imposed by disturbance. This is because a large fraction of genetic diversity is selectively neutral, and its distribution is therefore shaped by mutation, migration and genetic drift [44]. Indeed, even functional genetic variation can be strongly influenced by selectively-neutral demographic processes, such as population bottlenecks which can cause a loss of adaptive potential in colonising or remnant populations [45]. Key biological processes underlying neutral genetic patterns that can be affected by disturbance include mortality, reproduction, movement and social behaviour [21, 43, 46, 47]. In this review, we categorise effects of disturbance on demography as the direct effects, which relate to how disturbance directly imposes changes on biological processes such as mortality and reproduction, and indirect effects, relating to how species respond to the influence of disturbance on habitat suitability and landscape permeability. 


\section{Direct effects}

One prediction about the effects of disturbance events is that, when severe, they may cause a loss of genetic diversity within populations (in particular, allelic diversity or richness) when population sizes are reduced. Losses of allelic diversity and reduced expected heterozygosity within populations are anticipated where mortality is high and population recovery stems from a few in situ survivors or founding colonisers (particularly from a single source), and where ongoing immigration is low relative to the population growth rate (Fig. 1).

To illustrate the interacting influence of mortality and recruitment on the consequences of disturbance for within-population genetic diversity, we present a series of examples. The remarkably low genetic diversity in the Galapagos giant tortoise Chelonoidis nigra vandenburghi was attributed to a population bottleneck coinciding with a volcanic eruption causing high mortality in the sole, isolated population of this subspecies 100,000 years ago [28]. In cases such as this, the degree of loss of allelic diversity during population bottlenecks can be influenced by the duration of the bottleneck [48] and the spatial pattern of mortality (because alleles are rarely distributed in a random spatial pattern even within populations) [47]. Populations of the south-east Australian mallee emu-wren (Stipiturus mallee) also have low allelic richness because they are extirpated by regular fire and re-established by founder events driven by rare, stochastic colonisation (despite low ongoing migration between established populations) [19]. Other empirical studies have documented no losses of genetic diversity within disturbance-affected populations when survival has been high [49] or recovering populations recruit many individuals from multiple sources [31]. The reproductive strategies of some plants enable them to maintain high genetic diversity within populations even when disturbances like fire cause high mortality, because recruitment comes from seed banks established by pre-disturbance populations, and not from a small number of surviving individuals [50]. 
Disturbance also directly affects genetic differentiation among populations through its influence on genetic drift and migration. To illustrate, F $_{\text {ST }}$ among aquatic snail populations increased due to accelerated genetic drift following drought-induced reductions in local population size [51]. Conversely, FST among populations of the sailfin molly (Poecilia latipinna) decreased when a hurricane storm surge in coastal Florida increased 'migration' by directly transporting individual fish between populations [46]. Disturbance can often drive extinction-recolonisation dynamics in natural populations [52] resulting in at least two genetic scenarios. First, $\mathrm{F}_{\mathrm{ST}}$ can increase with the rate of population turnover where population recovery proceeds via colonisation by a small number of founders, followed by intrinsic population growth (Scenario 1) [53]. Conversely, population turnover may decrease FST where recolonisation and population growth occurs via high ongoing immigration, particularly from multiple sources (Scenario 2) [53, 54].

\section{Indirect effects}

As well as having direct effects on populations, disturbance can affect genetic diversity through its influence on the spatial and temporal patterns of habitat suitability and permeability [55]. Disturbance history at a point in the landscape determines its suitability as habitat for a species by influencing habitat structure, resource availability and community composition. Habitat dynamics that are driven by disturbance can influence how genetic diversity is distributed within and among populations because key biological processes like dispersal and reproduction develop in response to habitat patterns [56]. For instance, many species specialising in ephemeral habitat conditions (influenced by post-disturbance succession) exhibit high dispersal by both sexes consistent with the requirement for regular patch colonisation, and therefore have low genetic differentiation among populations $[19,57$, 58], even though fine-scale genetic structure can exist within patches [59]. 
In Box 1, we present a simulation framework to demonstrate how variation in the regimes and spatial patterns of disturbance in a landscape, coupled with simple ecological requirements of a species, can lead to strikingly different distributional patterns that vary over time and space. These simulations show that changes in fire patterns will affect the distribution of two Australian cool temperate forest species. Increasingly frequent fire (large or small) will reduce the landscape-wide distribution of mountain ash trees (Eucalyptus regnans), with flow-on effects for populations of the endangered Leadbeater's possum (Gymnobelideus leadbeateri) which depends on old, hollow-bearing mountain ash trees for shelter. This is because, in many places, frequently re-occurring fires will kill regenerating mountain ash trees before they reach reproductive maturity. A similar scenario in firesensitive populations of the shrub Persoonia mollis, in which frequent fire caused extinctionrecolonisation dynamics, was associated with low genetic diversity within populations and weak genetic differentiation between them [60].

In addition to influencing the spatial and temporal dynamics of habitat suitability, disturbance regimes can also influence the permeability of landscapes to dispersal [43, 61]. For instance, fire suppression changed forest structure in a way that reduced dispersal between populations of collared lizards (Crotaphytus collaris) inhabiting forest glades in the Missouri Ozarks [20]. Therefore, changes to disturbance regimes can influence the temporal and spatial configuration of suitable habitat. Such changes can cause demographic connectivity of populations to change, with implications for how genetic diversity is partitioned within and between populations. In order to make predictions about the neutral genetic impacts of changing disturbance regimes for particular species, we need to connect the direct and indirect demographic effects of disturbance. 
A key question for future research is whether we can predict the genetic consequences of changes to disturbance regimes for individual species. For Leadbeater's possum, as represented in Box 1, increasing the frequency of large fires may to lead to increased $F_{S T}$ with fire-driven population turnover (Scenario 1 as listed in the Direct effects section). This is because the number of colonisers to newly-suitable habitat will be low when patches of suitable habitat are large but geographically isolated (Box 1). If the fire frequency (at a point in the landscape) stays the same but fires are smaller, the number of recolonisation sources in the local neighbourhood is much greater because populations are smaller but less isolated (Box 1). Thus, FST is likely to decrease with population turnover (Scenario 2).

We suggest that species-specific predictive models could be framed around the interacting key processes including spatial and temporal variation in habitat suitability and landscape permeability, and the rates of dispersal, reproduction and mortality [62]. The former indirect processes set the constraints on distribution, abundance and movement across the landscape. The latter direct processes influence the severity and duration of population bottlenecks and the degree to which recovery occurs through survival or immigration, with the source and rate of arrival of immigrants or colonists being critical.

We also need to develop an understanding of how the key biological processes that mediate the genetic effects of disturbance vary through the stages of a disturbance regime [63]. For instance, fire stimulates immigration by some species [64] and reproduction in others [65], while other disturbances can drive shifts between sexual and clonal reproduction $[22,66]$. Furthermore, it may be critical to explore how disturbance influences the distribution of genetic variation measured at nuclear versus organellar DNA, because migration operates on these genomes in different ways. For instance, dispersal can differ between the sexes [67], or between seeds and pollen [50]. 


\section{How does disturbance influence genetic diversity through natural selection?}

Natural selection can potentially respond to a number of environmental and biotic processes associated with disturbance $[68,69]$. The effects of climatic instability on the morphology of Galapagos finches (Geospiza spp.) have provided evidence that disturbance events can cause temporal variation in the strength and direction of selection, leading to unpredictable patterns of evolution [70]. However, on particular timescales, disturbance does not necessarily equate to environmental instability because disturbances can be frequent and predictable occurrences within a long-term 'stable' environment [39]. Under such circumstances, local adaptation to disturbance regimes can occur [41]. For instance, topographically-mediated variation in fire regimes can influence spatial patterns of selection for fire response traits in plants, such as resprouting or obligate seeding [71]. Where traits conferring susceptibility or resilience to disturbance are phylogenetically conserved, selection imposed by disturbance regimes can alter the phylogenetic composition of communities [72]. Disturbance regimes can also have 'flow-on' evolutionary consequences; an experimental study on bacteria showed that the effects of repeated population bottlenecks on population density and genetic structure influenced the opportunity for kin selection and the evolution of cooperative biofilm-forming behaviour [12].

Although the examples provided above demonstrate that disturbance can influence selection on physiological or behavioural traits, we have less information on how and when genetic diversity itself is likely to be influenced by the selective effects of disturbance events and regimes. Nevertheless, we can make some predictions informed by theoretical and empirical research, and identify promising approaches for studying these questions. We hypothesise that disturbance-mediated variation in selection could cause temporal genetic patterns analogous to ecological succession (i.e. ‘temporal adaptation' [73]). In such cases, changes in allelic and genetic diversity could vary with disturbance frequency at loci under selection. We 
expect selection-driven responses to disturbance to be most apparent when disturbance events are large relative to the scale of dispersal (because migration can decrease local adaptation [74]) and when disturbance is frequent [41]. Lastly, we might expect ongoing feedbacks between the effects of changing disturbance regimes on demography and the functional genetic variation on which selection acts. For instance, experimental studies have shown that seagrass (Zostera marina) plots with higher genotypic diversity have greater resistance (survival) and resilience (recovery) to disturbance $[11,75]$. Thus, such populations are less likely to suffer losses of adaptive genetic diversity associated with population bottlenecks. Landscape genomics techniques offer exciting new opportunities to test hypotheses about selection-driven genetic changes in response to disturbance [76]. For instance, analyses of next-generation sequencing datasets of samples taken across landscapes might be used to distinguish the effects of disturbance-driven selection from those of demography on genomewide polymorphism data $[77,78]$. It will be important to determine whether we can detect variation in selection between the different temporal stages of a disturbance regime and between landscapes with different disturbance regimes. Theoretical and simulation research suggests that adaptive loci can respond rapidly to temporal variation in selective pressure, particularly when population differentiation is high [73, 79]. Landscape genomics studies aiming to test for selection-driven genetic responses to disturbance will need to use spatially and temporally replicated contrasts of sites with different disturbance histories to maximise the information provided by such studies. Furthermore, we stress the importance of replication in the design of such studies because false signals of selection can be generated by stochastic processes like accelerated genetic drift ('allele surfing'), at colonisation fronts or in rapidly expanding populations [80], which may be a frequent characteristic of the demography of disturbance-affected populations. 


\section{Can genetic analyses inform our understanding of population responses to disturbance?}

Our review has highlighted how disturbance influences genetic patterns at the individual and population levels [20,21], over short and long timescales [81, 82], and by selective and selectively-neutral processes $[46,83]$. We have discussed how the genetic effects of disturbance might be predictable using models framed around key ecological and demographic parameters. However, a major challenge for achieving this, and indeed for predicting the conservation implications of altered disturbance regimes, is that we often lack even a basic biological understanding of how species respond to disturbance [84]. Intuitively, genetic analysis itself can contribute to filling this knowledge gap in a number of ways. These include (a) elucidating demographic history associated with disturbance [81, 85], (b) determining the source and rate of recruitment to recovering populations [86], (c) identifying recolonisation routes [31], and (d) determining whether dispersal strategies differ between stable and recovering populations [87]. However, if we are to improve the application of genetic analyses in disturbance ecology, we need to identify the circumstances under which genetic analyses can inform our understanding of species' responses to disturbance, and when they cannot.

\section{Challenges and recommendations for the application of genetic analyses in disturbance} ecology

Populations in disturbance-prone ecosystems pose particular challenges for the application of genetic analyses to demographic and ecological research. First, such populations are characterised by temporal variation in abundance, reproduction and dispersal [20-22]. Because genetic diversity is influenced by both current and historical processes [88], it may be difficult to infer population processes from genetic patterns in landscapes with a history of disturbance. Second, multiple biological processes can generate similar genetic patterns [89]. This is because the ways in which we can quantify spatial and temporal patterns of genetic 
diversity (e.g. heterozygosity, allelic diversity, linkage disequilibrium, spatial genetic structure between individuals and populations) are far outnumbered by the interacting molecular, behavioural and ecological processes that generate the genetic patterns. This is particularly true in complex dynamic populations [53]. For instance, simulation modelling has shown that variable patterns of genetic structure among Florida sand skink (Plestiodon reynoldsi) populations with increasing time since fire might be due to changes in abundance, dispersal, or both [82]. In such circumstances, genetic data alone may be insufficient to resolve different biological hypotheses in disturbance-affected populations.

Below, we offer three recommendations for maximising the utility of genetic analyses for understanding the effects of disturbance on natural populations:

i. Where possible, use pre-and post-disturbance samples.

Genetic patterns are shaped by biological processes over a long temporal window [88]. Therefore, pre- and post-disturbance data will greatly increase the ability to identify the specific genetic changes associated with disturbance events [46] and, therefore, to use genetics to understand species' responses. For instance, residual animals, not immigrants, were identified as the source of recruits to bush rat (Rattus fuscipes) populations recovering from experimental disturbance by comparing their genotype likelihoods between predisturbance populations and candidate immigrant sources [86].

Where it is not possible to obtain pre- and post-disturbance samples, researchers should be aware of the temporal 'depth of signal' of the markers and analyses used. The sensitivity of a given genetic marker to demographic and evolutionary processes over different timescales will depend on the mode of inheritance and the process and rate of mutation [90]. Measures of genetic structure based on fine-scale spatial patterns of individual multilocus genotypes 
can be sensitive to processes occurring over the past few generations [67], whereas F $_{\mathrm{ST}}$ can retain genetic signals of processes occurring over hundreds of generations [88].

\section{ii. Take full advantage of non-genetic data.}

Where multiple biological scenarios can lead to the same genetic patterns, the use of additional data from other sources (abundance surveys, mark-recapture analyses, etc.) may refine our hypotheses, enabling genetic data to discriminate between competing explanations [91]. For instance, demographic and environmental data were used to establish a small set of plausible models of metapopulation dynamics of tropical freshwater snails [92]. Because these models led to alternative genetic predictions, the authors were then able to refine the understanding of metapopulation processes with genetic data.

\section{iii. $\quad$ Use realistic simulation models to aid biological inference.}

Increasing accessibility of spatially-explicit demographic and genetic simulation tools [67, 93] may be particularly valuable in disturbance research. Computer simulations can be used to evaluate the power of genetic analyses to resolve specific biological hypotheses by testing the genetic outcomes of variation in different biological and demographic parameters. They can also be used to evaluate whether cost and effort should be allocated to collecting and genotyping more samples or to generating a greater number of, or more variable, markers to answer specific questions $[67,94]$. Furthermore, simulations can contribute directly to the inference of biological processes by comparing the genetic predictions of alternative demographic simulation models with observed data. This can be done with post-hoc simulations to investigate the validity of biological conclusions [82], or within an approximate Bayesian computation framework to estimate biological and demographic parameters [95]. Such approaches, coupled with increasingly sophisticated methods for 
simulating disturbances in landscapes [96], will advance our understanding of the dynamics of genetic diversity in disturbance-prone landscapes.

\section{Concluding remarks}

Disturbance is an important ecological process but its influence on the distribution of genetic diversity has been largely overlooked. Recent research is beginning to show that disturbance can influence genetic diversity via biological and demographic processes, spatial and temporal variation in habitat suitability, and natural selection and evolution. Key areas for future research (summarised in Box 2) include the evaluation of genetic analyses for understanding demographic and evolutionary responses to disturbance, the impacts of changing disturbance regimes on the distribution of genetic diversity within and between populations, and the conservation and evolutionary relevance of these genetic impacts. Because the regimes of natural disturbances are changing, and the frequency of anthropogenic disturbances is increasing [2], it is imperative that we understand how these changes will impact biodiversity at its most fundamental level, genetic diversity.

\section{Acknowledgements}

Thanks to Sarah Brown and Luciano Beheregaray for discussions on the case studies presented, and to three anonymous reviewers and the Editor, Paul Craze, for constructive feedback on the manuscript. 


\section{References}

1 Sousa, W. (1984) The role of disturbance in natural communities. Annu. Rev. Ecol. Syst 15, $353-391$

2 Turner, M.G. (2010) Disturbance and landscape dynamics in a changing world. Ecology 91, 2833-2849

3 Banks, S.C., et al. (2011) Starting points for small mammal population recovery after wildfire: recolonisation or residual populations? Oikos $120,26-37$

4 Lindenmayer, D.B. and Tambiah, C.R. (2005) 2004 tsunami cleanup. Conserv. Biol. 19, 991-991

5 Connell, J.H. (1978) Diversity in tropical rainforests and coral reefs. Science 199, 13021310

6 Miller, A., et al. (2011) How frequency and intensity shape diversity-disturbance relationships. Proc. Natl. Acad. Sci. U. S. A. 108, 5643-5648

7 Amos, W., et al. (2001) The influence of parental relatedness on reproductive success. Proc. R. Soc. B-Biol. Sci. 268, 2021-2027

8 Keller, L.F. and Waller, D.M. (2002) Inbreeding effects in wild populations. Trends Ecol. Evol. 17, 230-241

9 Madsen, T., et al. (1999) Conservation biology - Restoration of an inbred adder population. Nature 402, 34-35

10 Hughes, A.R., et al. (2008) Ecological consequences of genetic diversity. Ecol. Lett. 11, 609-623

11 Reusch, T.B.H., et al. (2005) Ecosystem recovery after climatic extremes enhanced by genotypic diversity. Proc. Natl. Acad. Sci. U. S. A. 102, 2826-2831

12 Brockhurst, M.A., et al. (2007) Cooperation peaks at intermediate disturbance. Curr. Biol. $17,761-765$ 
13 Flannigan, M.D., et al. (2009) Implications of changing climate for global wildland fire. Int. J. Wildland Fire 18, 483-507

14 Westerling, A.L., et al. (2006) Warming and earlier spring increase western US forest wildfire activity. Science 313, 940-943

15 Williams, A.A.J., et al. (2001) The sensitivity of Australian fire danger to climate change. Clim. Change 49, 171-191

16 Wotton, B.M., et al. (2010) Forest fire occurrence and climate change in Canada. Int. J. Wildland Fire 19, 253-271

17 Cary, G.J., et al. (2009) Relative importance of fuel management, ignition management and weather for area burned: evidence from five landscape-fire-succession models. Int. J. Wildland Fire 18, 147-156

18 Lindenmayer, D.B., et al. (2011) Newly discovered landscape traps produce regime shifts in wet forests. Proc. Natl. Acad. Sci. U. S. A. 108, 15887-15891

19 Brown, S.M., et al. (2013) Limited Population Structure, Genetic Drift and Bottlenecks Characterise an Endangered Bird Species in a Dynamic, Fire-Prone Ecosystem. PLoS ONE 8, e59732

20 Templeton, A.R., et al. (2001) Disrupting evolutionary processes: the effect of habitat fragmentation on collared lizards in the Missouri Ozarks. Proceedings of the National Academy of Sciences 98, 5426-5432

21 Banks, S.C., et al. (2012) Adaptive responses and disruptive effects: how major wildfire influences kinship-based social interactions in a forest marsupial. Mol. Ecol. 21, 673-684

22 Travis, S.E., et al. (2004) Population structure and inbreeding vary with successional stage in created Spartina alterniflora marshes. Ecol. Appl. 14, 1189-1202

23 Manel, S., et al. (2003) Landscape genetics: combining landscape ecology and population genetics. Trends Ecol. Evol. 18, 189-197 
24 White, P.S. and Pickett, S.T.A. (1985) Natural disturbance and patch dynamics: an introduction. In The ecology of natural disturbance and patch dynamics (Pickett, S.T.A. and White, P.S., eds), pp. 3-13, Academic Press

25 Rossi, F., et al. (2007) Human trampling as short-term disturbance on intertidal mudflats: effects on macrofauna biodiversity and population dynamics of bivalves. Mar. Biol. 151, $2077-2090$

26 Sousa, W.P. (1979) Disturbance in intertidal boulder fields: the non-equilibrium maintenance of species diversity. Ecology 60, 1225-1239

27 Delmoral, R. and Bliss, L.C. (1993) Mechanisms of Primary Succession - Insights Resulting from the Eruption of Mount St-Helens. In Advances in Ecological Research, Vol 24, pp. 1-66, Academic Press Ltd

28 Gill, A.M. and Allan, G. (2008) Large fires, fire effects and the fire-regime concept. Int. J. Wildland Fire 17, 688-695

29 Lake, P.S. (2000) Disturbance, patchiness, and diversity in streams. J. North. Am. Benthol. Soc. $19,573-592$

30 Easterling, D.R., et al. (2000) Climate extremes: Observations, modeling, and impacts. Science 289, 2068-2074

31 Spear, S.F., et al. (2012) Genetic structure among coastal tailed frog populations at Mount St. Helens is moderated by post-disturbance management. Ecol. Appl. 22, 856-869 32 Simard, M., et al. (2012) What explains landscape patterns of tree mortality caused by bark beetle outbreaks in Greater Yellowstone? Glob. Ecol. Biogeogr. 21, 556-567

33 Zwolak, R. (2009) A meta-analysis of the effects of wildfire, clearcutting, and partial harvest on the abundance of North American small mammals. For. Ecol. Manage. 258, 539545 
34 Carnaval, A.C., et al. (2009) Stability Predicts Genetic Diversity in the Brazilian Atlantic Forest Hotspot. Science 323, 785-789

35 Keyghobadi, N. (2007) The genetic implications of habitat fragmentation for animals. Can. J. Zool. 85, 1049-1064

36 Lindenmayer, D.B., et al. (2009) Opportunity in the Wake of Natural "Disasters". Science $324,463-463$

37 Connell, J.H. and Slatyer, R.O. (1977) Mechanisms of succession in natural communities and their role in community stability and organization. Am. Nat. 111, 1119-1144 38 Swanson, M.E., et al. (2010) The forgotten stage of forest succession: early-successional ecosystems on forest sites. Frontiers in Ecology and the Environment 9, 117-125

39 Gill, A.M. (1975) Fire and the Australian flora: a review. Aust. For. 38, 4-25

40 Keeley, J.E. and Zedler, P.H. (2009) Large, high-intensity fire events in southern California shrublands: debunking the fine-grain age patch model. Ecol. Appl. 19, 69-94 41 Lytle, D.A. (2001) Disturbance regimes and life-history evolution. Am. Nat. 157, 525-536 42 Gill, A.M. and Bradstock, R. (1995) Extinction of biota by fires. In Conserving Biodiversity: Threats and Solutions (Bradstock, R.A., et al., eds), pp. 309-322, Surrey Beatty $\&$ Sons

43 Templeton, A.R., et al. (2011) The transition from isolated patches to a metapopulation in the eastern collared lizard in response to prescribed fires. Ecology 92, 1736-1747

44 Holderegger, R., et al. (2006) Adaptive vs. neutral genetic diversity: implications for landscape genetics. Landsc. Ecol. 21, 797-807

45 Dlugosch, K.M. and Parker, I.M. (2008) Founding events in species invasions: genetic variation, adaptive evolution, and the role of multiple introductions. Mol. Ecol. 17, 431-449 46 Apodaca, J.J., et al. (2013) Large-Scale Natural Disturbance Alters Genetic Population Structure of the Sailfin Molly, Poecilia latipinna. Am. Nat. 181, 254-263 
47 Vignieri, S.N. (2010) The genetic effects of ecological disturbance: flooding in jumping mice. Am. Nat. $175,126-135$

48 Garza, J.C. and Williamson, E.G. (2001) Detection of reduction in population size using data from microsatellite loci. Mol. Ecol. 10, 305-318

49 Suarez, N.M., et al. (2012) Genetic signature of a severe forest fire on the endangered Gran Canaria blue chaffinch (Fringilla teydea polatzeki). Conserv. Genet. 13, 499-507

50 Ayre, D.J., et al. (2009) Genetic structure of seedling cohorts following repeated wildfires in the fire-sensitive shrub Persoonia mollis ssp nectens. J. Ecol. 97, 752-760

51 Evanno, G., et al. (2009) Parallel changes in genetic diversity and species diversity following a natural disturbance. Mol. Ecol. 18, 1137-1144

52 Amarasekare, P. and Possingham, H. (2001) Patch dynamics and metapopulation theory: the case of successional species. J. Theor. Biol. 209, 333-344

53 Pannell, J.R. and Charlesworth, B. (2000) Effects of metapopulation processes on measures of genetic diversity. Philosophical Transactions of the Royal Society of London. Series B: Biological Sciences 355, 1851-1864

54 Roques, L., et al. (2012) Allee effect promotes diversity in traveling waves of colonization. Proc. Natl. Acad. Sci. U. S. A. 109, 8828-8833

55 Devictor, V. and Robert, A. (2009) Measuring community responses to large-scale disturbance in conservation biogeography. Divers. Distrib. 15, 122-130

56 Bowler, D.E. and Benton, T.G. (2005) Causes and consequences of animal dispersal strategies: relating individual behaviour to spatial dynamics. Biological Reviews 80, 205-225

57 Broyles, S.B. and Wyatt, R. (1993) Allozyme diversity and genetic structure in aouthern Appalachian populations of poke milkweed, Asclepias exaltata. Syst. Bot. 18, 18-30

58 Pierson, J.C., et al. (2010) Do male and female black-backed woodpeckers respond differently to gaps in habitat? Evol. Appl. 3, 263-278 
59 Pierson, J.C., et al. (2013) Breed Locally, Disperse Globally: Fine-Scale Genetic Structure Despite Landscape-Scale Panmixia in a Fire-Specialist. PLoS ONE 8, e67248

60 Krauss, S.L. (1997) Low genetic diversity in Persoonia mollis (Proteaceae), a firesensitive shrub occurring in a fire-prone habitat. Heredity 78, 41-49

61 Spear, S.F. and Storfer, A. (2010) Anthropogenic and natural disturbance lead to differing patterns of gene flow in the Rocky Mountain tailed frog, Ascaphus montanus. Biol. Conserv. $143,778-786$

62 Schrey, A.W., et al. (2011) Fire Alters Patterns of Genetic Diversity Among 3 Lizard Species in Florida Scrub Habitat. J. Hered. 102, 399-408

63 Chung, M.Y., et al. (2007) Effects of population succession on demographic and genetic processes: predictions and tests in the daylily Hemerocallis thunbergii (Liliaceae). Mol Ecol $16,2816-2829$

64 Muona, J. and Rutanen, I. (1994) The short term impact of fire on the beetle fauna in boreal forests. Ann. Zool. Fenn. 31, 109-111

65 Pausas, J.G., et al. (2004) Plant functional traits in relation to fire in crown-fire ecosystems. Ecology 85, 1085-1100

66 Xie, Z.W., et al. (2001) Clonality in wild rice (Oryza rufipogon, Poaceae) and its implications for conservation management. Am. J. Bot. 88, 1058-1064

67 Banks, S.C. and Peakall, R. (2012) Genetic spatial autocorrelation can readily detect sexbiased dispersal. Mol. Ecol. 21, 2092-2105

68 Hairston, N.G., et al. (2005) Rapid evolution and the convergence of ecological and evolutionary time. Ecol. Lett. 8, 1114-1127

69 Kokko, H. and Lopez-Sepulcre, A. (2007) The ecogenetic link between demography and evolution: can we bridge the gap between theory and data? Ecol. Lett. 10, 773-782 
70 Grant, P.R. and Grant, B.R. (2011) Causes of lifetime fitness of Darwin's finches in a fluctuating environment. Proc. Natl. Acad. Sci. U. S. A. 108, 674-679

71 Clarke, P.J. (2002) Habitat islands in fire-prone vegetation: do landscape features influence community composition? Journal of Biogeography 29, 677-684

72 Helmus, M.R., et al. (2010) Communities contain closely related species during ecosystem disturbance. Ecol. Lett. 13, 162-174

73 Blanquart, F. and Gandon, S. (2013) Time-shift experiments and patterns of adaptation across time and space. Ecol. Lett. 16, 31-38

74 Blanquart, F., et al. (2012) The effects of migration and drift on local adaptation to a heterogeneous environment. J. Evol. Biol. 25, 1351-1363

75 Hughes, A.R. and Stachowicz, J.J. (2004) Genetic diversity enhances the resistance of a seagrass ecosystem to disturbance. Proc. Natl. Acad. Sci. U. S. A. 101, 8998-9002

76 Narum, S.R., et al. (2013) Genotyping-by-sequencing in ecological and conservation genomics. Mol. Ecol. 22, 2841-2847

77 Allendorf, F.W., et al. (2010) Genomics and the future of conservation genetics. Nat Rev Genet 11, 697-709

78 Schoville, S.D., et al. (2012) Adaptive Genetic Variation on the Landscape: Methods and Cases. Annual Review of Ecology, Evolution, and Systematics 43, 23-43

79 Landguth, E.L. and Balkenhol, N. (2012) Relative sensitivity of neutral versus adaptive genetic data for assessing population differentiation. Conserv. Genet. 13, 1421-1426

80 Excoffier, L., et al. (2009) Genetic Consequences of Range Expansions. Annual Review of Ecology, Evolution, and Systematics 40, 481-501

81 Beheregaray, L.B., et al. (2003) Genes record a prehistoric volcano eruption in the Galapagos. Science 302, 75-75 
82 Schrey, A.W., et al. (2010) Fire increases variance in genetic characteristics of Florida Sand Skink (Plestiodon reynoldsi) local populations. Mol. Ecol. 20, 56-66

83 DiBattista, J.D., et al. (2011) Anthropogenic disturbance and evolutionary parameters: a lemon shark population experiencing habitat loss. Evol. Appl. 4, 1-17

84 Driscoll, D.A., et al. (2010) Fire management for biodiversity conservation: Key research questions and our capacity to answer them. Biol. Conserv. 143, 1928-1939

85 Steinfartz, S., et al. (2007) Genetic impact of a severe El Nino event on Galapagos marine iguanas (Amblyrhynchus cristatus). PLoS ONE 2, e1285

86 Peakall, R. and Lindenmayer, D. (2006) Genetic insights into population recovery following experimental perturbation in a fragmented landscape. Biol. Conserv. 132, 520-532 87 Simmons, A.D. and Thomas, C.D. (2004) Changes in dispersal during species' range expansions. Am. Nat. 164, 378-395

88 Landguth, E.L., et al. (2010) Quantifying the lag time to detect barriers in landscape genetics. Mol. Ecol. 19, 4179-4191

89 Double, M.C., et al. (2005) Dispersal, philopatry, and infidelity: Dissecting local genetic structure in superb fairy-wrens (Malurus cyaneus). Evolution 59, 625-635

90 Sunnucks, P. (2000) Efficient genetic markers for population biology. Trends Ecol. Evol. $15,199-203$

91 Lowe, W.H. and Allendorf, F.W. (2010) What can genetics tell us about population connectivity? Mol. Ecol. 19, 3038-3051

92 Lamy, T., et al. (2012) Testing metapopulation dynamics using genetic, demographic and ecological data. Mol. Ecol. 21, 1394-1410

93 Estoup, A., et al. (2012) Estimation of demo-genetic model probabilities with Approximate Bayesian Computation using linear discriminant analysis on summary statistics. Mol. Ecol. Resour. 12, 846-855 
94 Ryman, N. and Palm, S. (2006) POWSIM: a computer program for assessing statistical power when testing for genetic differentiation. Mol. Ecol. Notes 6, 600-602

95 Csillery, K., et al. (2010) Approximate Bayesian Computation (ABC) in practice. Trends Ecol. Evol. 25, 410-418

96 Keane, R.E., et al. (2004) A classification of landscape fire succession models: spatial simulations of fire and vegetation dynamics. Ecol. Model. 179, 3-27

97 Excoffier, L., et al. (1992) Analysis of molecular variance inferred from metric distances among DNA haplotypes: application to human mitochondrial DNA restriction data. Genetics $131,479-491$

98 Smouse, P.E. and Peakall, R. (1999) Spatial autocorrelation analysis of individual multiallele and multilocus genetic structure. Heredity $82,561-573$

99 McCarthy, M.A., et al. (1999) Fire regimes in mountain ash forest: evidence from forest age structure, extinction models and wildlife habitat. For. Ecol. Manage. 124, 193-203

100 Bradstock, R.A., et al. (2005) Which mosaic? A landscape ecological approach for evaluating interactions between fire regimes, habitat and animals. Wildl. Res. 32, 409-423

101 Cary, G.J. and Banks, J.C.G. (2000) Fire regime sensitivity to global climate change: An Australian perspective. Biomass Burning and Its Inter-Relationships with the Climate System $3,233-246$

102 King, K.J., et al. (2013) Contrasting fire responses to climate and management: insights from two Australian ecosystems. Global Change Biology 19, 1223-1235

103 Midgley, G.F., et al. (2010) BioMove-an integrated platform simulating the dynamic response of species to environmental change. Ecography 33, 612-616 


\section{Glossary}

Allelic diversity: The number of different alleles per locus in a population. Allelic richness is a measure of allelic diversity standardised for sample size.

Disturbance intensity: Power of disturbing agent at a point (e.g. energy output from fire in $\mathrm{W} \mathrm{m} \mathrm{m}^{-2}$ or fireline intensity in $\mathrm{kW} \mathrm{m}^{-1}$ ).

Disturbance regime: Temporal pattern of disturbance events, usually at a point, including frequency, intensity, season and type of disturbance.

Disturbance severity: Magnitude of impact of disturbing agent on above and below ground organic matter (e.g. extent of canopy tree scorch in a fire).

Ecological disturbance: "any relatively discrete event in time that disrupts ecosystem, community, or population structure and changes resources, substrate availability, or the physical environment" [24].

$\boldsymbol{F}_{\text {ST: }}$ A measure of genetic differentiation describing the proportion of genetic variation among populations relative to that within populations. $F_{\mathrm{ST}}$ ranges from zero (no differentiation) to one (complete differentiation).

Genetic differentiation: A measure of how genetic diversity is partitioned among populations within a species $\left(\right.$ e.g. $\left.F_{\mathrm{ST}}\right)$

Genetic diversity: Variation in genetic material from an individual, population or species. This can be characterised by multiple statistics, typically calculated at the locus level. These statistics include observed heterozygosity within individuals. At the population level, common measures include allelic diversity, allelic richness, observed and expected heterozygosity. Among-population level genetic diversity is typically represented by measures of genetic differentiation such as $\mathrm{F}_{\mathrm{ST}}$ and its analogues, often within the framework 
of Analysis of Molecular Variance, which allows hierarchical partitioning of genetic variance [97].

Genetic drift: Random changes in allele frequencies between generations due to chance alone. Genetic drift is stronger in small than in large populations because of the increased chance of sampling errors.

Genetic structure: Non-random distribution of genetic diversity that can form over space or time. Spatial patterns of genetic structure can be measured as genetic differentiation between populations (e.g. FST) or by fine-scale spatial analyses of genotypes within populations [98].

Heterozygosity: Commonly measured as observed or expected heterozygosity. Observed heterozygosity $\left(\mathrm{H}_{\mathrm{O}}\right)$ is the average observed heterozygosity of individuals at the population level. $\mathrm{H}_{\mathrm{E}}$ is the expected heterozygosity of individuals within populations under the assumptions of Hardy-Weinberg equilibrium.

Landscape genetics / landscape genomics: Research fields which combine population genetics (or genomics), landscape ecology and spatial statistics to quantify spatial variation at neutral or selection-driven loci in relation to heterogeneity in physical or biotic environmental conditions.

Mean return interval: Average time between disturbance events

Metapopulation: Part of the spectrum of spatial dynamics where local populations occur in discrete habitat, have asynchronous dynamics, are sustained for at least a few generations, and where some populations become extinct and others are recolonised by dispersing individuals.

Neutral vs selection-driven genetic variation: Neutral genetic variation is not influenced by natural selection, whereas selectively-driven genetic variation has an adaptive advantage or disadvantage and is thus influenced by selection. 
Population bottleneck: A rapid and severe reduction in population size that can cause a rapid loss of genetic diversity, which can be accentuated by genetic drift if the population size reduction persists for multiple generations.

Succession: Temporal change in community composition after disturbance. 


\section{Box 1. Effects of disturbance regimes on spatial population dynamics}

Approach: We use simplified models to demonstrate that variation in the spatial and temporal patterns of disturbance affects the distribution and dynamics of natural populations, with implications for patterns of genetic diversity and differentiation.

Case studies: We illustrate the approach with case studies of a forest plant and animal species affected by fire: mountain ash (Eucalyptus regnans), the world's tallest flowering plant, and Leadbeater's possum (Gymnobelideus leadbeateri), an endangered arboreal marsupial dependent on hollow-bearing mountain ash trees for shelter.

1 Habitat suitability: We established a habitat suitability index for each species. These were

2 defined as simple binary indices (Fig. I) according to two key dimensions of fire occurrence 3 at points in the landscape, time since fire (TSF) and the most recent inter-fire interval (IFI)

4 [39]. We assumed an otherwise uniform landscape. More realistic extensions could include variables related to disturbance type, intensity or season, and other ecological characteristics including topography, climate or vegetation heterogeneity.

7 Disturbance simulations: We generated fire patterns on a 150x150 cell landscape, varying 8 frequency (mean inter-fire interval 100 years or 50 years in each cell) and the size of fires 9 (50x50 cells or $5 \times 5$ cells). In the Victorian Central Highlands, where our case study species co-exist, the typical regime of large stand-replacing fires involves an inter-fire interval of 75150 years [99]. We simulated fires over 1000 years and recorded the TSF and most recent IFI for each cell at the end of the simulations.

Predicted habitat suitability maps: We combined the simulated fire histories with the habitat suitability criteria (Fig. I) to predict the distribution of suitable habitat for each species at year 1000 of each simulation (Fig. II). This combination of simple models reveals that variation in spatial and temporal patterns of disturbance can lead to important differences in 
17 the dynamics of species' distributions [100]. Changing the frequency and spatial scale of fires

18 influenced the overall amount of suitable habitat for each species, as well as the size and

19 spatial configuration of suitable habitat patches, which can have important implications for

20 landscape-wide genetic patterns.

21 Extensions: Although our case study features fire as a disturbance, the approach is

22 extendable to other disturbance types. It can be extended to more realistic environmental and

23 population scenarios by modelling underlying environmental variation influencing habitat

24 suitability and disturbance [101, 102]. The approach could also include key biological

25 processes such as dispersal and mortality [103], to provide a demographic context for making

26 predictions about the genetic consequences of disturbance. 


\section{Box 2. Key outstanding questions}

What is the power of genetic analysis to elucidate population responses to disturbance?

What can genetic analyses tell us that we cannot learn about disturbance in other ways?

Do different combinations of disturbance, demography and species traits lead to distinctive genetic patterns?

Can we distinguish the roles of different population processes in driving changes in genetic diversity for particular species and disturbance types?

What supporting data (abundance, dispersal, etc.) are most informative for refining our inference from genetic data?

How can we take full advantage of next-generation sequencing technology and computational simulation modelling approaches to improve our understanding of the biotic effects of disturbance from genetic data?

What are the genetic impacts of long-term disturbance regimes?

What are the cumulative effects of the long-term sequence of disturbance-driven demographic changes on genetic diversity?

How do disturbance regimes influence genetic diversity through their influence on spatial and temporal patterns of habitat suitability?

How does natural selection influence the genetic effects of disturbance events and regimes? 
To what extent does disturbance history account for the contemporary distribution of genetic diversity across landscapes? Can the genetic effects of past disturbances obscure those of contemporary population processes?

What is the conservation relevance of the genetic effects of disturbance?

Do genetic changes resulting from severe or increasingly frequent disturbance reduce the capacity to adapt to other environmental changes? What will be the long-term evolutionary consequences of changing disturbance regimes? Does anthropogenic disturbance cause the loss of genetic diversity and thereby reduce the resilience of species to disturbance?

Do long-term disturbance regimes cause feedbacks between the demographic and genetic effects of disturbance? For example, do disturbance-induced bottlenecks cause a loss of genetic diversity, thereby increasing the susceptibility of populations to future

60 disturbances? 


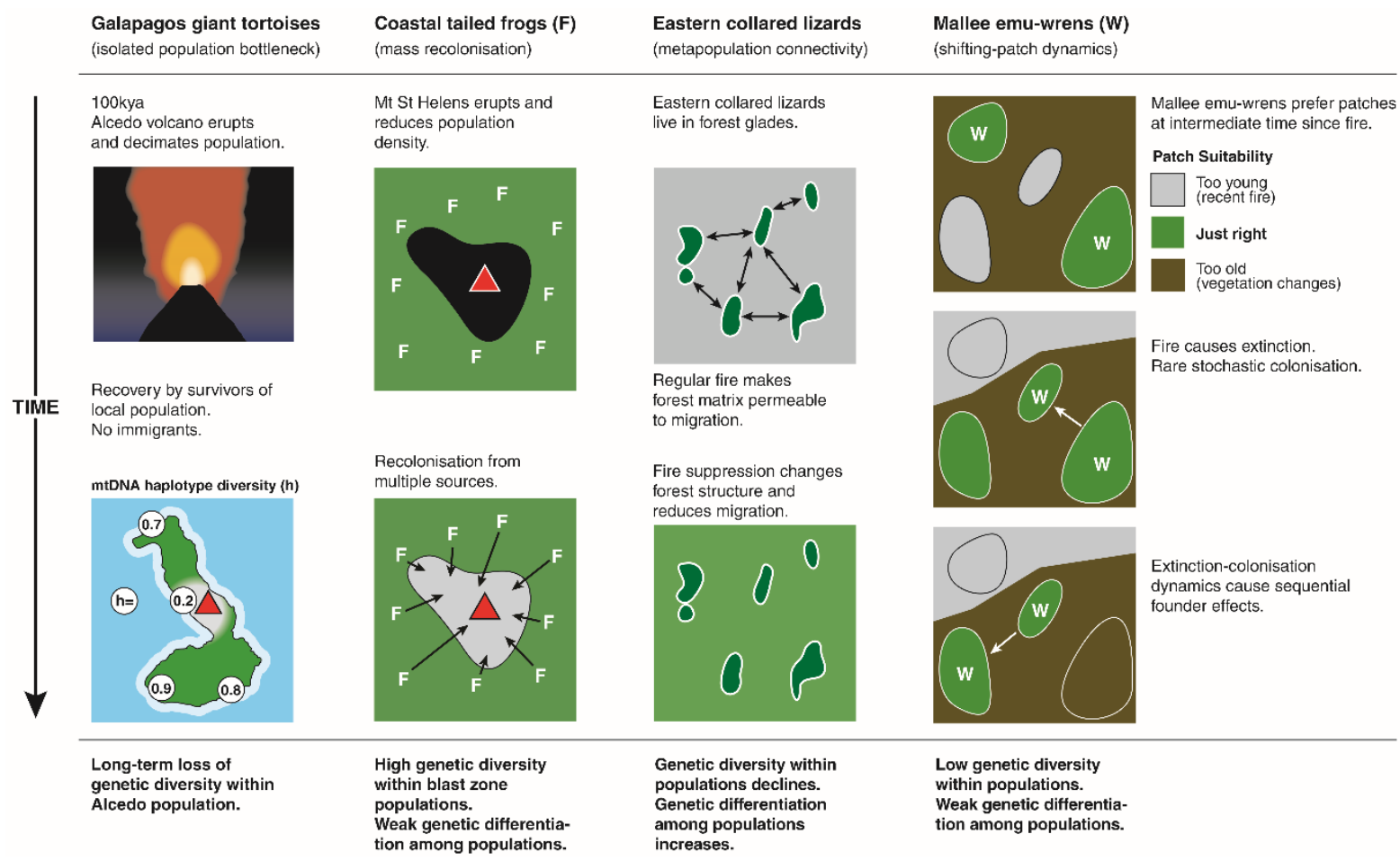

Figure 1. Contrasting demographic and genetic responses to disturbance. A set of case

65

66

67

studies featuring contrasting genetic outcomes of disturbance events and regimes, and how they affect mortality, migration and habitat suitability. The two case studies on the left (Galapagos giant tortoises [81] and coastal tailed frogs [31]) contrast the neutral genetic consequences of disturbance events causing major mortality in an isolated and connected population, respectively. The case studies on the right (Eastern collared lizards [20, 43] and mallee emu-wrens [19]) show how disturbance regimes affect habitat permeability and habitat suitability, respectively, thereby influencing genetic diversity and differentiation through their effects on spatial population dynamics. Illustration by Clive Hilliker, Fenner School of Environment \& Society, ANU. 
TSF

a.

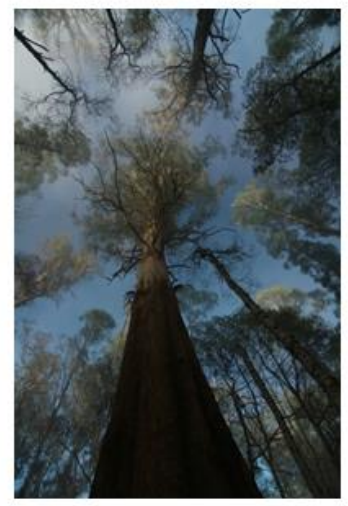

b.

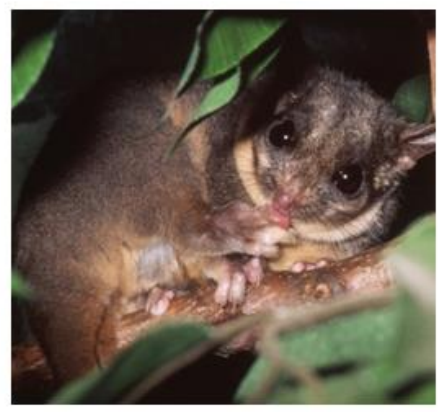

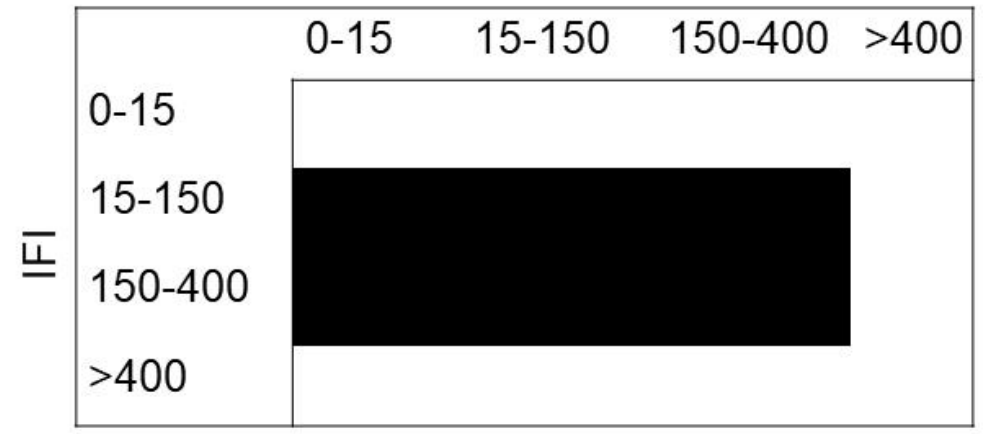

TSF

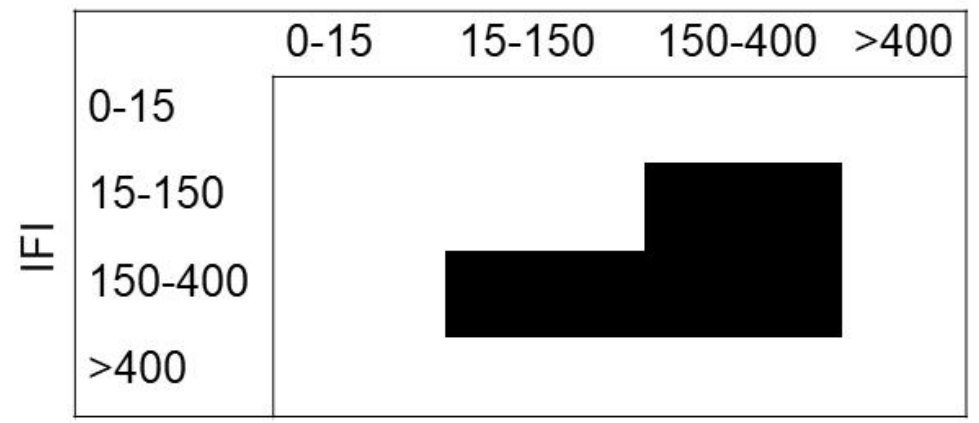

(Box 1) Figure I. Habitat suitability indices. Simple binary habitat suitability indices for

mountain ash (top) and Leadbeater's possum (bottom) defined as suitable or unsuitable depending on the most recent inter-fire interval and the time in years since the last fire. Mountain ash grows in south-eastern Australia and is killed by severe fires, after which it regenerates from seed. It becomes reproductively mature after 15-20 years and begins to senesce from 150-400 years. In the absence of fire, mountain ash stands are commonly replaced by cool temperate rainforest. Leadbeater's possum is dependent on decayed hollowbearing trees for shelter in mountain ash forest. Suitable hollows begin to form in trees that are at least 150 years old. Leadbeater's possum can persist in young forest stands after fires that occur in old forest, because hollow-bearing trees killed by fire can remain standing for decades. Photos: David Blair (mountain ash), David Lindenmayer (Leadbeater's possum). 


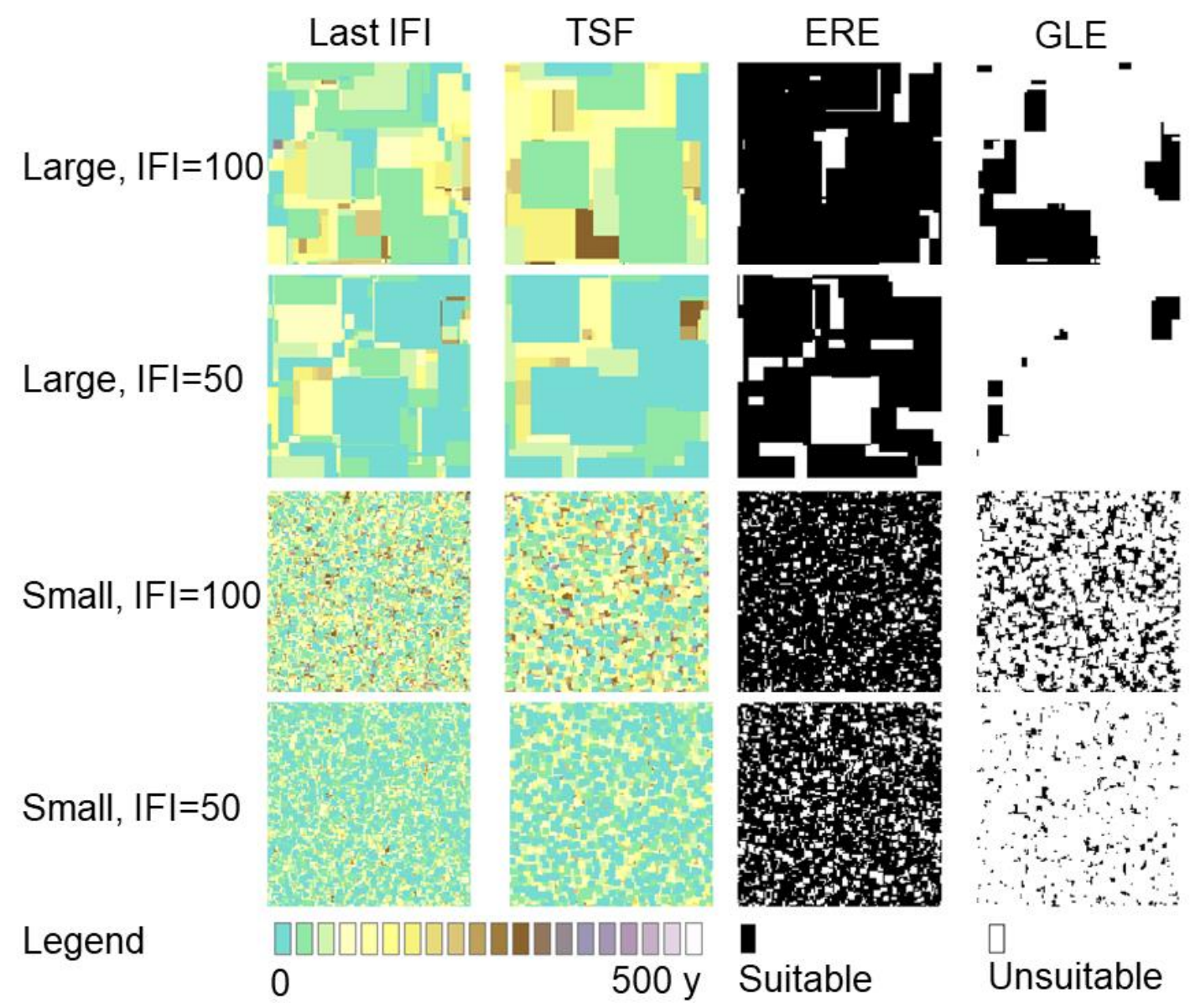

(Box 1) Figure II. Fire simulations and predicted distributions. Simulated fire histories, including most recent inter-fire interval (IFI) and time since last fire (TSF), and resulting

91 habitat distributions for mountain ash trees (Eucalyptus regnans: ERE) and Leadbeater's

92 possum (Gymnobelideus leadbeateri: GLE) under four different spatial patterns and regimes

93 of fire. Large (50x50 cells) and small ( $5 \times 5$ cells) were simulated with a mean fire return

94 interval (per cell) of 100 or 50 years. Species distributions were predicted according to the habitat suitability criteria from Fig. 1. 